\title{
Strategies to Address Substance-Abusing and Substance-Impaired Healthcare Professionals
}

\author{
Anna M. Smith, DBA \\ Walden University, Minneapolis, Minnesota, United States \\ Douglas G. Campbell, PhD \\ Walden University, Minneapolis, Minnesota, United States \\ (iD https://orcid.org/0000-0002-3989-1571
}

Contact: douglas.campbell@mail.waldenu.edu

\begin{abstract}
This research explored strategies used by some healthcare leaders to address substance-abusing and impaired healthcare professionals. The conceptual framework for this qualitative case study was the concept of due diligence. Data were collected from 40 managers and supervisors, three executives, and company documents, and analyzed to reveal elements of a comprehensive strategy. A effective comprehensive strategy consists of policies that address the causes, the short-term and long-term aspects, and both the ethical and legal aspects. Policies and procedures are equally applied, are easily accessible, are well communicated, are supported by training, provides positive encouragement, provides a path for recovery, includes audits to ensure compliance and provides for organizational reviews to determine the need for updating. Leaders who implement a such a strategy to address substance abuse among healthcare professionals can create positive social change by ensuring a safer environment for patients, employees, and their communities they serve.
\end{abstract}

Keywords: substance-impaired; substance-abuse; doctors; nurses; healthcare

Date Submitted: April 25, 2019 | Date Published: June 2, 2020

\section{Recommended Citation}

Smith, A. M., \& Campbell, D. G. (2020). Strategies to address substance-abusing and substance-impaired healthcare professionals. International Journal of Applied Management and Technology, 19, 97-106. https://doi.org/10.5590/IJMAT.2020.19.1.08

\section{Introduction}

Between $8 \%$ and $15 \%$ of doctors, nurses, and pharmacists have abused or misused drugs or alcohol in the workplace (Bryson, 2017; Kunyk, 2015; Oreskovich et al., 2015). Healthcare professionals who practice or perform duties while impaired by alcohol or other chemical agents, place patients at risk of harm, and they jeopardize the reputation and financial health of the healthcare organization they work for or with (Georgiou, 2013, Kunyk, 2015; Fusion, 2014). Despite the evidence of the prevalence of workplace substance abuse and substance impairment among healthcare professionals and its negative effects on healthcare organizations, there is scant research to inform healthcare leaders of possible strategies to more effectively address these problems. 


\section{Literature Review}

More than 100 publications from multiple disciplines were reviewed for this research. These included a wide range of seminal works, peer-reviewed publications, and authoritative statistics and governmental reports. The reviewed literature revealed a complex relationship between organizational leadership, management strategies, and individual substance abuse and substance impairment. Different concepts, definitions, and explanations of substance abuse and substance impairment existed in the literature. Little of the reviewed literature directly addressed the effectiveness of management strategies used by some healthcare leaders to address substance-abusing and substance-impaired healthcare professionals in the workplace. However, there was a significant amount of literature that documented the extensive nature of the problem and resulting consequences to organizations and patients of healthcare professionals practicing while substance impaired.

The epidemiology of the impaired healthcare professional is broad, affecting all genders, socioeconomic status, educational background, culture and geographic location (Rundio, 2013). Competency is the hallmark of the healthcare profession and is requisite for the health, safety, and welfare of patients (Burton, 2014). The American Hospital Association (2013) described safe nursing and medical care as a distinguishing characteristic of healthcare organizations. Accrediting organizations such as the Hospital Consumer Assessment of Healthcare Providers and System Organizations financially penalize hospitals failing to provide safe patient care. The healthcare industry is concerned about substance impairment as it potentially diminishes competency and decreases the ability of the healthcare professional to practice according to accepted professional standards. Substance impairment results from substance use, abuse, or dependency (Georgiou, 2013).

\section{Method}

\section{Purpose, Research Question, Population, Design, and Framework}

The purpose of this qualitative multiple case study was to explore strategies used by some leaders of healthcare organizations to address substance-abusing and substance-impaired healthcare professionals in the workplace. The central research question was this:

Research Question: What management strategies do some healthcare leaders use to address substance-abusing and substance-impaired healthcare professionals in the workplace?

The research population consisted of 40 managers and supervisors of healthcare professionals in the same hospital, owned by a major healthcare organization that owns several hospitals in the United States. This healthcare organization, and this hospital in particular, had an exemplary reputation for medical care delivery.

The participating hospital managers and supervisors received a qualitative questionnaire, and three executive leaders were interviewed using a semistructured interview process. Additionally, a variety of organizational documents were reviewed for relevant information. With respect to the practical application of this research, it presented an opportunity to enhance the depth, breadth and understanding of how healthcare leadership need to address substance-impaired healthcare professionals in the workplace, which in turn has important benefits, because such insight can shape future organizational initiatives related to the substance abuse workplace issue.

The conceptual framework for this research was the concept of due diligence, both the ethical and legal aspects. Due diligence refers to the care a reasonable person should take before entering into an agreement or 
a transaction with another party (Violette \& Shields, 2007). The concept of due diligence applies to organizations and leaders who seek to provide a safe and effective workplace environment. The basic concept of due diligence in this context extends the idea of safe practice linking healthcare leaders, healthcare professionals, and healthcare organizations. Figure 1 presents the interrelationships of the ethical and legal aspects of due diligence as applicable to healthcare leaders.

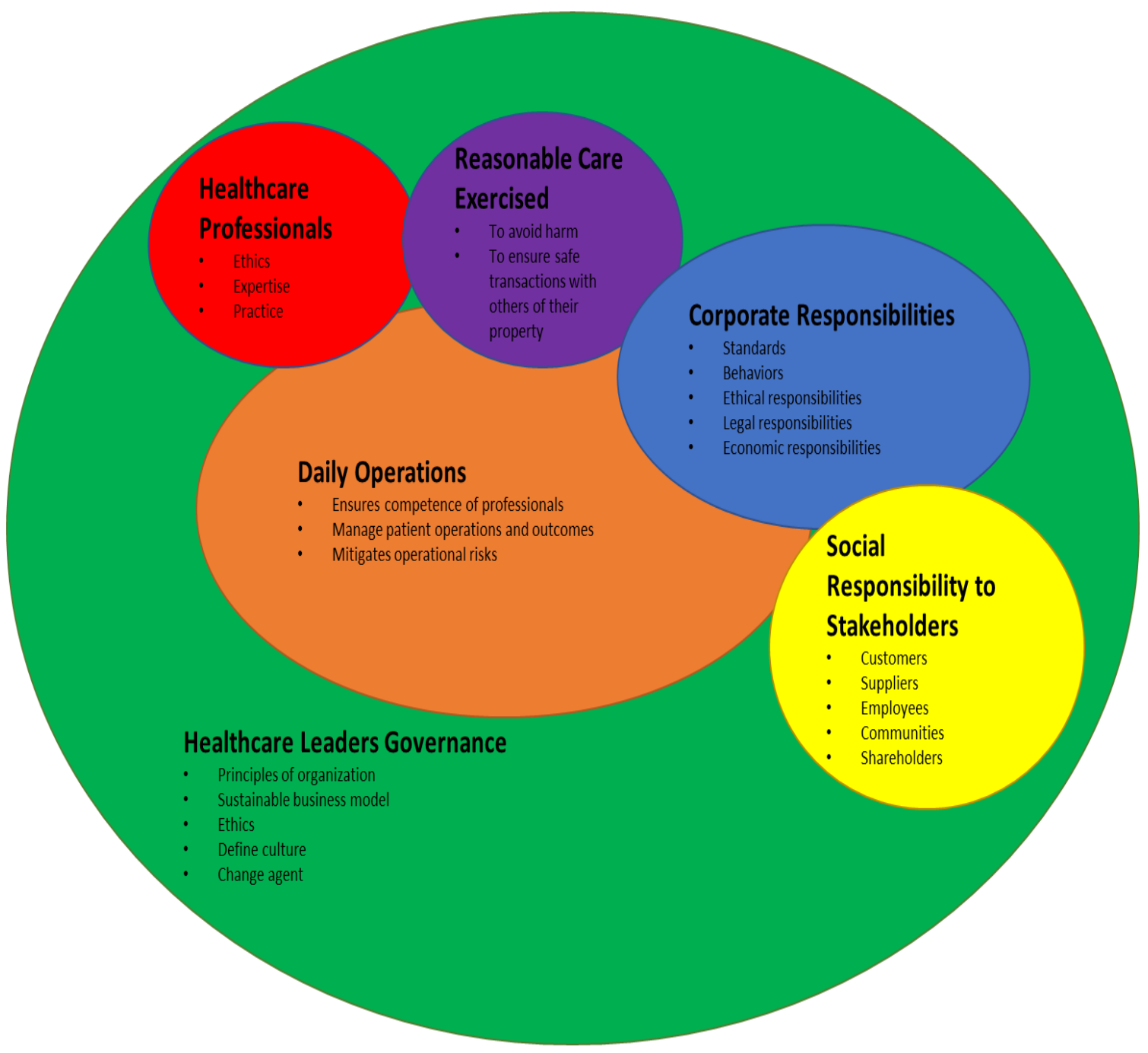

Figure 1. Due Diligence Conceptual Application to Healthcare Leaders

\section{Data Collection Questions}

The following is the questionnaire completed by the participating managers and supervisors:

1. What management strategies do you use when addressing the known or suspected substanceimpaired healthcare professional?

2. Describe your preparation as a leader regarding the problem of substance-impaired healthcare professional.

3. Describe the management strategy or process for drug testing the suspected impaired healthcare professional in your organization who is actively providing patient care. 
4. What management strategies do you use to support a healthcare professional enrolled in a substance abuse recovery program?

5. What management strategies do you use to monitor a substance-impaired healthcare professional returning to the work environment?

6. What additional information would you like to add?

The following are the interview questions asked of participating executive leaders:

1. What management strategies do you use when addressing the known or suspected to be impaired nurse or physician?

2. What methods do you use for educating managers and supervisors on strategies when addressing the impaired nurse or physician?

3. What strategies do you use to manage reentry into practice for healthcare professionals after completing a substance impairment treatment program?

4. What additional information would you like to add?

\section{Data Reliability, Validity, and Analysis}

Significant efforts were made to ensure the reliability of the data. Member checking ensured the accuracy of the interpretation of the participants' statements. This enhanced accuracy, prior to subjecting the transcripts to formal coding and analysis, contributed to validity. Integrity was enhanced by the use of different data sources and data collection techniques. Appropriate analytical procedures were followed to provide consistency. Such consistency helped control for possible researcher bias.

A computer software program was useful for organizing the data, coding, counting frequencies of words, querying keywords, and identifying relationships and patterns in the data. Descriptive codes were identified from the raw data based on the concepts found in the conceptual framework and literature review. The process of data analysis in qualitative research involves the evaluation of the resulting data by working with the data, breaking the data down, synthesizing data, and searching for themes or patterns to discover the critical or relevant data elements (Freeman et al., 2015). Methodological triangulation was accomplished using all the data sources. Based on the pattern of the data sets, key themes were identified. These themes were compared to the reviewed literature, and then viewed through the lens of the concept of due diligence. The findings of this research are specific to the healthcare community. Transferability of qualitative research to a different setting can be difficult and may require a broader context (Keane et al., 2013). Therefore, the judgment of the reader and the findings of future researchers will eventually determine the transferability of the findings of this research.

\section{Findings}

The themes derived from the data along with various perspectives from participants revealed that the leaders, managers and supervisors all recognized the serious need to address the problems of substance-abusing and substance-impaired healthcare professionals in the workplace, and also the inadequacy of the current efforts to protect patients from substance-impaired doctors and nurses. Additionally, all participants expressed concerns about the dangers of working with substance-impaired individuals. Executive participants' responses included the words safety, patient harm, apprehensive, concern, and at-risk. Managers and supervisors frequently used the words stress, concern, diversion, substance-abuse, training, education, and staff safety. These differences in language seem to indicate that the executives focused more on the risk to the organization as a whole, whereas the managers and supervisors focused more on how the staff were affected. 
Despite the participating healthcare organization's exemplary reputation, the analysis of the data revealed a mere façade of policies and procedures that masked a surprising degree of dis-functionality. Therefore, the six themes that emerged from the data reflect the organization's challenges with crafting and implementing consistent, fair, and appropriate policies that address the substance-impaired healthcare professional and provide enhanced staff and patient protection. The six themes were: (a) selective application of policy, (b) managerial training on the indications of substance impairment, (c) monitoring and surveillance support, (d) policies for reentry into work environment, (e) synergistic integration between work and life, and (f) managerial training on the legal and ethical implications of reporting.

\section{Theme 1: Selective Application of Policy}

Despite the organization's published substance-abuse policies and procedures, the managers and supervisors expressed frustration with the availability, the communication and the inconsistent application of those policies and procedures. The data revealed that among the managers and supervisors, their knowledge and understanding of published substance-abuse policies significantly varied. Additionally, managers and supervisors stated that the organization's policies and procedures were inconsistently followed and sometimes ignored. Specifically, compliance depended on the individuals on a particular work shift. Some participants expressed concern that substance abuse policies for nurses and other employees, were different from those policies for physicians. Physicians were considered contract labor, not employees of the hospital, and thus supposedly required different polices. Participants explained that the policies were electronically stored, sometimes difficult to access, and not always appropriately updated. Our review of the organization's policies and procedures regarding substance abuse and substance impairment confirmed that they were stored in separate locations and not readily accessible to managers and supervisors on duty.

In contrast, the executive participants expressed confidence with the state of the policies and confidence that they were uniform, accessible to all staff, and being consistently and appropriately applied. As a result, there was no ongoing effort to address the above stated concerns of the managers and supervisors. Valdes (2014) concluded that a state of inconsistent knowledge and inconsistent application of substance abuse policies and procedures does not promote quick intervention and corrective action.

\section{Theme 2: Managerial Training on the Indications of Substance Impairment}

All participants agreed that there was a need for enhanced continuing professional education to ensure that executives, managers, supervisors and other employees can recognize the diverse and sometimes subtle indications of possible substance abuse, substance-impairment and stress-related behaviors that can lead to substance abuse. This observation is consistent with Banja (2014), who emphasized the need for consistent education regarding signs and symptoms of substance impairment. Banja further specified that when employees think their manager and supervisors know how to detect substance abuse they are more likely to do something about it. Therefore, the potential for substance use at work decreases.

\section{Theme 3: Monitoring and Surveillance Support}

Healthcare organizations are expected to provide resources such as workplace health policies which include the signs and symptoms of substance abuse and substance impairment (Kinkle, 2015). Participants agreed that well designed continuing education is vital in recognizing the diverse and often subtle signs and symptoms of substance abuse or impairment. However, managers and supervisors described how the organization's training was insufficient, and as a result indications of substance abuse or impairment were often not identified, or not identified early enough to prevent patient and organizational harm. 
The National Institute for Drug Abuse (2014) concluded that any healthcare facilities with controlled substances on the premises are at risk of those drugs being stolen by substance abusers. While participants acknowledge that there had been illegal drug diversion events within their organization, they also described in positive terms the organization's substance control and surveillance and monitoring systems, noting the new technology was helpful in preventing the illegal diversions of substances. Additionally, some participants emphasized the need to continue developing better quality assurance processes, and of the importance of oversight audits when a drug diversion event does occur.

\section{Theme 4: Policies for Reentry Into Work Place}

Some healthcare professionals who successfully complete treatment of substance-abuse are allowed to return to the workforce (O’Neil \& Cadiz, 2014). All the participants stressed the importance of ensuring that such individuals are fully recovered and healthy before being allowed to resume medical duties and access to narcotics. Managers and supervisors commented that every situation is unique, and they described how ultimately the returning individual is accountable for the success or failure of their reentry journey. Managers and supervisors supported random drug testing as a way of monitoring for relapse. However, participants' responses were inconsistent regarding how this would occur in their organization, who would be responsible for the testing, how long or how frequently drug testing should be performed, and who should have knowledge of the results. These questions are not well addressed in the organization's current published policies and procedures.

\section{Theme 5: Synergistic Integration Between Work and Life Balance}

The managers and supervisors agreed that the stressful work environment of the hospital contributed to some healthcare professionals abusing drugs and alcohol and to being impaired in the workplace. In their opinions, such factors as long work hours, excessive overtime, heavy patient work assignments, understaffing, and the lack of peer compassion contribute to poor job satisfaction, low morale, poor work-life balance, and incidents of substance abuse. The findings of Shanafelt et al. (2015) also indicated that an excessively stressful work environment does contribute to the excessive use of narcotics and alcohol by healthcare providers-also, that physicians, not interns, were less likely to tolerate high workloads and the effects on their personal life.

\section{Theme 6: Managerial Training on the Legal and Ethical Implications of Reporting}

Many managers and supervisors admitted to being apprehensive about reporting suspected substanceimpaired healthcare professionals. Their apprehensiveness was tied to their concerns about the fairness of the process, the ethics of reporting and the potential risks to themselves of reporting. Some of these participants were uncertain when and how reporting should occur per the organization's policy. Apprehension was evident even among those participants who said that they were quite familiar with the written policies. Some participants said that they would keep "their suspicion to themselves." New (2013) found that feelings of loyalty, guilt, and fear often prevented employees from reporting other employees. These same feelings of ethical dilemma were evident in some of the participants. New also found a correlation between years of experience and their comfort level with reporting a suspicious colleague. Additionally, James (2013) and Pronovost and Wachter (2013) found that healthcare managers and supervisors who were confident in the reporting process were quicker to report the suspected substance-impairment. 


\section{Discussion}

\section{Conclusions}

The findings of this case study revealed how healthcare leaders, with the best of intentions, implement policies and procedures to address the substance-impaired healthcare professional in the workplace, yet fail to meet the ethical and legal standards of due diligence. Publishing policies and procedures represent the first step, but not a comprehensive strategy to ensure due diligence. A comprehensive strategy consist of well thought out policies and procedures that (a) address the causes of the problem, (b) address the short-term and long-term aspects of the problem, (c) address both the ethical and legal aspects of the problem, (d) are equally applied, (e) are easily accessible, (f) are well communicated, (g) are supported by initial training and a program of continuous training, (h) provide positive encouragement for employees and contractors to adhere to the policies and procedures, (i) provide a path for recovery, and (j) include audits to ensure compliance and organizational reviews to determine when policies and procedures should be updated.

\section{Recommendations}

The first steps in addressing any problem is to obtain a full understanding of the problem, the identification of the causes, finding the systemic failures to address the problem and acceptance of responsibility for fixing the problem. Healthcare leaders should make a sustained effort to understand the problem and the evolution of the problem, and the long- and short-term aspects of the problem. Addressing the problem of healthcare professionals abusing substances and being impaired in the workplace requires a comprehensive strategy that must consist of far more than simply publishing reporting and disciplinary policies. Healthcare leaders should cultivate understanding and sensitivity to both the ethical and legal aspects of the problem and their responsibilities.

Substance abuse by healthcare professionals is driven by working and operating in an environment that is emotionally and physically stressful, and sometimes where substance abuse is tacitly facilitated by a culture of tolerance, excuses and negligent oversight. Leaders should strive to institute a healthcare business model that is financially sustainable while providing a sustainable healthy environment for patients and internal and external stakeholders. Development of a work and life integration program with supportive measures would greatly contribute to the evolution of a caring and supportive work culture throughout the organization.

Published policies and procedures should be reviewed, and if necessary rewritten, to ensure they are comprehensive, humane, equally applicable to all healthcare professionals, easily understood and easily executed. The policies and procedures should not be open to interpretation, yet they should be flexible enough to provide clear and sufficient direction and due diligence in all areas of the medical facility, and for all eventualities. All these policies and procedures should be easily and immediately accessible from all areas of the medical facility and by all employees.

Communicating the policies and procedures to employees is of the utmost importance. Simply having employees read the policies and procedures, while important, is not sufficient to ensure that they have the degree of knowledge and familiarity with them that dramatically increases the likelihood of compliance. An education plan is needed that imparts both knowledge of and understanding of the problem and resulting dangers, and knowledge and understanding of the policies and procedures and how to access them for review or reference. Managers and supervisors should receive additional and continuing training on the application and use of the policies and procedures.

The early identification of potential substance abusers or of substance impairment is critical to protecting patients, staff and the organization. Priority should be given to the development and implementation of a 
specific program of training on the signs and indications of likely substance abuse and substance impairment. This training should occur quarterly and be updated as new information becomes available. All employees should be ethically encouraged to report any suspicions that they might have, assured that their prompt action will be appreciated by the management team and that they will be protected from any possible negative consequences. Incorporating a drug reporting telephone hot line with a dedicated number facilitates prompt reporting.

There should be humane and nonpunitive provisions for healthcare professionals who seek treatment for substance abuse and addiction. An increased emphasis on identifying substance abusers along with the nonpunitive option for assistance may encourage substance-abusing healthcare professionals to seek treatment. Provisions should also be made for fully recovered individuals to return to the job under a continuing regime of increased oversight and testing to ensure the individual does not return to using or abusing any substance.

Diversions of drugs from workplace storage and from patients are a facilitator of substance abuse and a result of substance addiction. Diverting drugs can also be a source of considerable illegal revenue for the immoral employees. There should be a comprehensive strategy for the provision of monitoring and surveillance resources which support the early detection of drug diversions. The most current technology should be employed, such as AI, retina or finger scanning. Such systems can provide synchronized institutional usage and access to narcotics. Suspicious transactions should be investigated immediately to promote a culture of safety and compliance. Leaders should ensure managers and supervisors receive training related to accessing, interpreting and trending data from these systems. Managers and supervisors should receive ongoing training regarding appropriate narcotic storage, dispensing and disposal procedures. Reports generated from these systems should be disseminated to managers and supervisors. Leaders should ensure that managers and supervisors receive adequate training on accessing, interpreting and trending data from these systems.

To send a clear message that substance abuse, substance impairment and drug diversions will not be tolerated, and that appropriate response will occur, those managers and supervisors that report such events should receive feedback on what was done to address their report. Without such feedback managers and supervisors are left wondering if their report was accurate, whether it was taken seriously and whether anything was done to address the event. Closing the feedback loop and giving information to managers and supervisors regarding the actions taken increases confidence in the leadership of the organization.

In this research, we documented the executives lacked awareness of how poorly the organization's substance abuse and substance impairment policies and procedures were being communicated, were understood and being followed. When the executives are not aware of problems, then those problems are not addressed by the organization in an appropriate manner. There should be a regime of continuing internal and external audits, done by independent auditors, of the functioning of and compliance with substance abuse and substance impairment policies and procedures. There should be an annual, comprehensive organizational review of the effectiveness of the strategies in place, followed by the implementation of a plan for the needed corrective actions and improvements. This will require executives to be intimately familiar with the organization's policies and procedures and other details of the comprehensive organizational strategy to address substance abuse, substance impairment and drug diversions.

\section{The Significance of the Study}

The dissemination and implementation of a comprehensive strategy to address the problem of the substanceabusing and substance-impaired healthcare professionals may prevent the adverse outcomes to patients and lessen the interruption of appropriate healthcare operations. Understanding how leaders should address the substance-impaired nurse or physician may promote a productive and safe work environment. Additionally, 
the recommendations derived from this research could undergird positive social change by improving the dissonance of conflicting attitudes, beliefs or behaviors in managing the impaired healthcare professional. Positive social change is a deliberate process of creating and applying strategies and actions to promote the worth, dignity, and development of individuals, communities, organizations, and institutions (Kinkle, 2015). Healthcare leaders who recognize the cascading ill-toward effects of substance impairment can promote positive social change by ensuring a safe environment for patients, employees and the community.

\section{Limitations, Possible Weakness, and Recommendations for Further Research}

Qualitative research is inherently an exploration of a phenomenon, and data comes from capturing peoples' perspectives concerning that phenomenon. Therefore, no formal measurements of outcomes were employed in this research, and this can be considered a limitation. It could also be argued that the limited population of this multiple case study could mean that the findings might have limited transferability. However, the organization that was studied is a major regional medical healthcare provider with multiple locations and employs policies and procedures that are common throughout the healthcare industry. We recommend that researchers pursue further understanding of how peer behavior may enable or discourage substance abuse and substance impairment by healthcare professionals.

\section{Final Thoughts}

Healthcare organizations are an integral part of creating healthy communities. Healthcare leaders have the responsibility to protect patients, to support staff and to maintain high standards of care throughout the organization (Berge, \& Lanier, 2014; Georgiou, 2013). Substance-abusing and substance-impaired nurses or doctors are more prevalent than most people care to think about. It is an uncomfortable subject, but one so important that by avoiding it people are endangered, lives are damaged, and the financial viability of healthcare organizations are put at risk. Addressing the problems of substance abuse and substance impairment among nurse and doctors is a substantial task, but it is not an impossible task if there is a sincere will to get the job done.

\section{References}

American Hospital Association. (2013). Patient safety. http://www.aha.org

Banja, J. (2014). Alcohol and drug testing of health professionals following preventable adverse events: A bad idea. The Journal of Bioethics, 1(2), 25-36. https://doi.org/10.1080/152651.2014.964873

Berge, K. H., \& Lanier, W. (2014). Bloodstream infections outbreaks related to opioid-diverting healthcare workers: A cons-benefit analysis of prevention and detection. Mayo Clinic Proceedings, 89(7), 866868. https://doi.org/10.1016/jmayocp2014.04.10

Bryson, E. O. (2017). The opioid epidemic and the current prevalence of substance use disorder in anesthesiologists. Anesthesia and Medical Disease, 31(3), 388-339. https://doi.org/10.1097/ACO.0000000000000589

Burton, K. L. (2014). Emerging from the darkness and stepping into the light: Implementing an understanding of the experience of nurse addiction into nursing education. Journal of Nursing Education \& Practice, 4(1), 151-164. https://doi.org/10.5430/jnep.v4n4p151

Freeman, M., Gregen, K., \& Josselson, R. (2015). The promises of qualitative inquiry. American Psychologist, $70(2), 1-9$. https://doi.org/10.103.1037/a0038597

Fusion, J. (2014, January 20). Problems associated with drug \& alcohol abuse in the workplace. http://smallbusiness.chron.com/problems-associated-drug-alcohol-abuse-workplace-1238.html 
Georgiou, M. (2013). Alcoholism and unemployment: A panel data analysis for the Industrial world. SSRN Working Paper Series. https://doi.org/10.2139/ssrn.2220227

James, J. T. (2013). A new evidence-based estimate of patient harms associated with hospital care. Journal of Patient Safety, 9(3), 122-128. https://doi.org/10.1097/PTS.obo13e3182948a69

Keane, S., Lincoln, M., \& Smith, T. (2013). Retention of allied health professionals in rural New South Wales: A comparison of public and private practitioners. BMC Health Services Research, 12(1), 1-22. https://doi.org/10.1186/1472-6963-13-32

Kinkle, S. A. (2015). Identifying substance use disorder in nursing. Nursing Management, 12(5), 53-54. https://doi.org/10.1097/01NUMA.0000473512.38679.ca

Kunyk, D. (2015). Substance use disorders among registered nurses: Prevalence, risks, and perceptions in a disciplinary jurisdiction. Journal of Nursing Management, 23(2), 54-64. https://doi.org/10.1111/jonm.12081

Mandela, N. (2010). Conversations with myself. Farrar, Straus, and Giroux. https://www.nelsonmandela.org/content/page/a-selection-of-nelson-mandela-quotes

National Institute for Drug Abuse. (2014). Controlled Substance Act Part B, 21U.S.C. http://www.drugabuse.gov/

New, K. (2013). Institutional diversion, prevention, detection, and response. National Council of State Boards of Nursing. https://www.ncsbn.org/0613 DISC Kim New.pdf

O'Neill, C., \& Cadiz, D. (2014). Worksite monitors protect patients from unsafe nursing practices. Journal of Nursing Regulation, 5(2), 16-21. https://doi.org/0.1016/52155-8256(15)30079X

Oreskovich, M. R., Shanafelt. T., Dyrbye, L.N., Tan, L., Sotile, W., Satele, D., \& Boone, S. (2015). The prevalence of substance use disorders in American physicians. American Journal of Addiction, 24(1), 30-38. https://doi.org/10.1111/ajad.12173

Pronovost, P., \& Wachter, R. M. (2013). Progress in patient safety: A glass fuller than it seems. American Journal of Medical Quality, 29(2), 165-169. https://doi.org/10.1177/1062860613495554

Rundio, A. (2013). Peer assistance for nurses with substance-use disorders. Journal of Nursing Clinics of North America, 48(4), 459-463. https://doi.org/10.1016/j.cnur.2013.05.002

Shanafelt, T., Hasan, O., Dyrbye, L. N., Sinsky, C., Satele, D., Sloan, J., \& West, C. P. (2015). Changes in burnout and satisfaction with work-life balance in physicians and the general U.S. working population between 2011 and 2014. Mayo Clinic Proceedings, 90, 1600-1613. https://doi.org/10.1016/j.mayocp.2015.08.023

Valdes, J. A. (2014). The concept of reentry in the addicted anesthesia provider. American Association of Nurse Anesthetist Journal, 82(2), 95-100.

Violette, G., \& Shields, J. (2007). Aids in the hiring process. The CPA Journal, 77(3), 64-65.

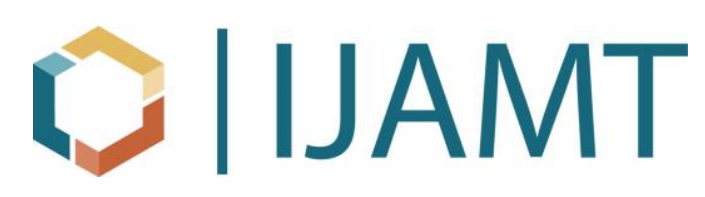

The International Journal of Applied Management and Technology (IJAMT), sponsored by Walden University's College of Management and Technology, is a peer-reviewed, online journal that addresses contemporary national and international issues related to management and technology. 